\title{
The relation of endocan and galectin-3 with ST-segment resolution in patients with ST-segment elevation myocardial infarction
}

\author{
Yaşar Turan ${ }^{A-F}$, Vahit Demir ${ }^{A-F}$ \\ Department of Cardiology, Faculty of Medicine, Bozok University, Yozgat, Turkey \\ A - research concept and design; $\mathrm{B}$ - collection and/or assembly of data; $\mathrm{C}$ - data analysis and interpretation; \\ $D$ - writing the article; $E$ - critical revision of the article; $F$ - final approval of the article
}

Address for correspondence

Vahit Demir

E-mail: dr.vdemir@hotmail.com

\section{Funding sources}

This study was funded by the Scientific Research Projects of Bozok University in Yozgat, Turkey (2015TF/A203).

\section{Conflict of interest}

None declared

\section{Acknowledgements}

The authors would like to thank the Scientific Research Projects of Bozok University in Yozgat, Turkey, and paramedics in the study area.

Received on May 20, 2019

Reviewed on February 10, 2020

Accepted on February 10, 2020

Published online on April 28, 2020

Cite as

Turan Y, Demir V. The relation of endocan and galectin-3 with ST-segment resolution in patients with ST-segment elevation myocardial infarction. Adv Clin Exp Med. 2020;29(4):453-458. doi: 10.17219/acem/118126

DOI

10.17219/acem/118126

\section{Copyright}

Copyright by Author(s)

This is an article distributed under the terms of the

Creative Commons Attribution 3.0 Unported (CC BY 3.0)

(https://creativecommons.org/licenses/by/3.0/)

\begin{abstract}
Background. ST-segment elevation myocardial infarction (STEMI) remains a leading cause of morbidity and mortality around the world. In patients with STEMI undergoing primary percutaneous coronary intervention (PPCI), electrocardiographic measures of ST-segment resolution (STR) may give information about the myocardial perfusion and poor prognosis.

Objectives. To investigate the relation of endocan and galectin-3 levels with STR in STEMI patients.

Material and methods. In this cross-sectional study, 98 consecutive patients undergoing PPCI for STEMI were enrolled. Synergy between percutaneous coronary intervention with taxus and cardiac surgery (SYNTAX) scores were recorded. Electrocardiograms were assessed at baseline and $60 \mathrm{~min}$ after PPCI. According to STR levels, patients undergoing PPCI $(n=98)$ were divided into complete STR group $(\geq 70 \%, n=53)$ and incomplete STR group $(<70 \%, n=45)$.
\end{abstract}

Results. Serum glucose, total cholesterol, low-density lipoprotein cholesterol, SYNTAX score, endocan and galectin-3 levels were significantly higher and ejection fraction was significantly lower in the incomplete STR $(<70 \%)$ group ( $p<0.05$ for all). Body mass index (BMI) $(p=0.046)$ and galectin-3 $(p=0.037)$ were independently associated with the SYNTAX score. Endocan $(p=0.044)$ and galectin-3 $(p=0.017)$ were independent predictors of incomplete STR.

Conclusions. In patients with STEMI, the levels of endocan and galectin-3 may be helpful in identifying patients with a higher risk of insufficient myocardial perfusion and worse clinical outcome after PPCl.

Key words: ST-segment elevation myocardial infarction, galectin-3, endocan, ST-segment resolution 


\section{Introduction}

ST-segment elevation myocardial infarction (STEMI) is a form of coronary artery disease (CAD) that requires immediate and adequate attention to avoid deadly consequences. It may occur as a result of coronary thrombosis in an epicardial coronary artery, which is damaged by a chronic and inflammatory process known as atherosclerosis. ${ }^{1}$ The high mortality rate of STEMI could be offset by providing sufficient blood flow to the damaged myocardium as quick as possible. ${ }^{2}$ Primary percutaneous coronary intervention (PPCI) has become the more preferred treatment option for STEMI, over the past 2 decades, compared to thrombolysis.

Primary percutaneous coronary intervention aims to restore complete blood flow in the infarcted arteries in STEMI patients. However, good blood flow does not always indicate an effective microvasculature perfusion at the myocyte level. ${ }^{3}$ In other words, good angiographic blood flow is unfortunately not always accompanied by good clinical outcomes. ST-segment deviations may be good indicators of the myocyte physiology, and thus the preferable option is to determine the success of PPCI electrocardiographically (ECG) by measuring ST-segment resolution (STR). ${ }^{4}$ Previous data suggests that a sufficient STR may be predictive for clinical outcomes in STEMI patients undergoing PPCI. ${ }^{4,5}$

Endocan or endothelial cell-specific molecule-1 is synthesized in the vascular endothelium and gives information about angiogenesis and endothelial cell functions. ${ }^{6}$ Endocan plays a key role in cell adhesion and is reported to be associated with endothelial dysfunction. ${ }^{7}$ In patients with STEMI, higher endocan levels on admission were reported to be associated with a worse cardiovascular outcome and high synergy between percutaneous coronary intervention with taxus and cardiac surgery (SYNTAX) score. ${ }^{8}$

Galectin-3 is a $\beta$-galactoside-binding lectin expressed by activated macrophages that regulates inflammation, ventricular remodeling and fibrosis. ${ }^{9}$ In addition, galectin-3 was also reported to have a prognostic value in patients with CAD, possibly related to its role in plaque destabilization. ${ }^{10}$ Moreover, an association between higher levels of galectin-3 and a lower ejection fraction, as well as higher mortality, was reported in patients diagnosed with acute coronary syndrome. ${ }^{11}$

The specific pathophysiology of endocan and galectin-3's association with negative cardiovascular outcomes remains to be elucidated. The assessment of the predictors of incomplete STR in acute phase might be helpful in identifying the patients at high risk for ineffective coronary reperfusion. Aggressive treatment may be planned for these patients to recover sufficient coronary perfusion.

This study evaluated the concentrations of endocan and galectin-3 in patients with STEMI and determined the relation of these parameters with STR.

\section{Methods}

\section{Study population}

Ninety-eight patients with STEMI with symptoms for less than $12 \mathrm{~h}$ who had underwent coronary angiography and PPCI at the Department of Cardiology of Bozok University in Turkey were prospectively enrolled in this cross-sectional study. ST-segment elevation myocardial infarction diagnosis was based on the presence of prolonged chest pain and ST-segment elevation (in all leads other than V2-V3: $\geq 1 \mathrm{~mm}$, in leads V2-V3: $\geq 2 \mathrm{~mm}$ in men $\geq 40$ years; $\geq 2.5 \mathrm{~mm}$ in men $<40$ years, or $\geq 1.5 \mathrm{~mm}$ in women regardless of age). ${ }^{12}$ Exclusion criteria were the following: cardiogenic shock, ventricular tachycardia, ventricular fibrillation, thrombolysis within the last $24 \mathrm{~h}$, oral anticoagulant therapy, indication for emergency coronary artery bypass grafting, active severe bleeding, uncontrolled hypertension, severe renal failure, and left bundle branch block. Routine treatment was initiated according to current STEMI guidelines. Information regarding risk factors, including age, gender, diabetes mellitus, hypertension, family history, and smoking status was obtained. Weights and heights of participants were measured. Body mass index (BMI) was calculated as (weight in kilograms)/(height in meters) ${ }^{2}$. Transthoracic echocardiography was performed for each patient immediately after admission using a commercially available machine (Philips Logic Affiniti 50G machine; Philips, Amsterdam, Netherlands) with a broadband transducer. The study was conducted according to the Declaration of Helsinki. The ethics committee of Bozok University approved the study and informed consent was obtained from all patients.

\section{Electrocardiogram}

A standard 12-lead ECG was recorded in all patients at admission (baseline ECG) and 60 min after the completion of revascularization with PPCI. All ECGs were interpreted by an observer who was unaware of other patient data. ST-segment elevation in millivolt was calculated $20 \mathrm{~ms}$ after the J point. The sum of ST-segment elevations in leads I, aVL, and V1-V6 for noninferior infarction and in leads II, III, aVF, V5, and V6 for inferior infarction were calculated separately. ST-segment resolution was calculated by reducing the sum of the ST-segment elevation from the baseline ECG to the 60-min ECG and was expressed as a percentage. A decrease in the sum of ST-segment elevation by at least $70 \%$ was categorized as complete STR. Patients were divided into 2 groups according to the degree of STR: $<70 \%$ (incomplete resolution) and $\geq 70 \%$ (complete resolution).

\section{Coronary angiography}

The SYNTAX score is the most commonly used anatomical scoring system worldwide to assess the complexity 
of coronary lesions. ${ }^{13}$ The baseline coronary angiograms were evaluated to determine the SYNTAX score by an experienced interventional cardiologist unaware of the patients' clinical or laboratory results. The SYNTAX score was determined for all coronary lesions with $>50 \%$ diameter stenosis in a vessel $>1.5 \mathrm{~mm}$ based on SYNTAX score calculator 2.1 (www.syntaxscore.com).

\section{Laboratory measurements}

Samples were taken from the antecubital vein when the patients were admitted to the hospital. Serum endocan and galectin-3 levels have been shown to be higher in patients with a myocardial infarction than in the healthy population. ${ }^{14,15}$ Although the entire study group consisted of patients with an acute myocardial infarction, blood samples were collected as soon as possible at the first admission to the hospital, to minimize possible changes resulting from the myocardial infarction. The venous blood samples were drawn from the patients at admission to the hospital. The blood samples used for endocan and galectin-3 measurements were centrifuged and preserved in the freezer $\left(-80^{\circ} \mathrm{C}\right)$. Enzyme-linked immunosorbent assay (ELISA) kit (YH-Biosearch, Shanghai, China) was used for measuring serum endocan and galectin-3 levels.

\section{Statistical analysis}

Data was analyzed using PASW Statistics for Windows v. 18.0 (SPSS Inc., Chicago, USA). Continuous variables were reported as mean \pm standard deviation (SD ) or range (min-max). Normally distributed variables were compared with Student's t-test, whereas non-normally distributed variables were compared with Mann-Whitney U test. Categorical variables were reported as percentages and counts and compared using the $\chi^{2}$ test. Correlation of the SYNTAX score with study parameters was assessed by Pearson or Spearman's correlation analysis as appropriate.

A multivariate logistic regression analysis was used to identify the predictors of incomplete STR. Furthermore, a linear regression analysis was performed to find predictors of SYNTAX score in the study population. Variables that had $\mathrm{p}<0.2$ in the univariate analysis were included in the multivariable models. For all statistical tests, $\mathrm{p}<0.05$ was considered significant.

\section{Results}

Patients' demographic characteristics and baseline clinical data are presented in Table 1. According to STR levels,

Table 1. Demographic and clinical data of the participants

\begin{tabular}{|c|c|c|c|}
\hline Characteristics & $\begin{array}{l}\text { Incomplete STR }<70 \% \\
(\mathrm{n}=45)\end{array}$ & $\begin{array}{l}\text { Complete STR } \geq 70 \% \\
(n=53)\end{array}$ & $\mathrm{p}$-value \\
\hline Age [years] & $62.5 \pm 10.6$ & $59.1 \pm 10.3$ & 0.097 \\
\hline Gender, n (female/male) & $9 / 36(25)$ & $8 / 45(18)$ & 0.354 \\
\hline BMI $\left[\mathrm{kg} / \mathrm{m}^{2}\right]$ & $28.74 \pm 4.93$ & $29.15 \pm 4.62$ & 0.678 \\
\hline Hypertension, n (\%) & $19 / 45(42)$ & $23 / 53(47)$ & 0.535 \\
\hline Diabetes mellitus, n (\%) & $16 / 45(35)$ & $15 / 53(28)$ & 0.290 \\
\hline Smoking, n (\%) & $24 / 45(53)$ & $25 / 53(47)$ & 0.343 \\
\hline Family history, n (\%) & $17 / 45(37)$ & $14 / 53(28)$ & 0.228 \\
\hline Systolic blood pressure [mm Hg] & $120.6 \pm 20.2$ & $119.2 \pm 18.6$ & 0.737 \\
\hline Diastolic blood pressure [mm Hg] & $74.3 \pm 12.6$ & $73.5 \pm 12.0$ & 0.740 \\
\hline Heart rate $[b p m]$ & $75.8 \pm 12.5$ & $74.1 \pm 13.1$ & 0.422 \\
\hline Initial troponin level [ng/mL] & $13.211(18-50.000)$ & $9.169(11-50.000)$ & 0.574 \\
\hline Peak troponin level [ng/mL] & $38.843(19.900-50.000)$ & $33.244(11.900-50.000)$ & 0.142 \\
\hline Serum glucose [mg/dL] & $145(87-524)$ & $118(60-557)$ & 0.019 \\
\hline Serum creatinine $[\mathrm{mg} / \mathrm{dL}]$ & $0.91 \pm 0.21$ & $0.88 \pm 0.24$ & 0.422 \\
\hline Total cholesterol [mg/dL] & $204.9 \pm 42.2$ & $180.7 \pm 43.6$ & 0.007 \\
\hline Triglyceride [mg/dL] & $141(73-798)$ & $115(71-379)$ & 0.063 \\
\hline $\mathrm{HDL}-\mathrm{C}[\mathrm{mg} / \mathrm{dL}]$ & $37.04 \pm 7.2$ & $39.68 \pm 8.1$ & 0.137 \\
\hline LDL-C [mg/dL] & $136.3 \pm 35.2$ & $114.6 \pm 37.5$ & 0.004 \\
\hline SYNTAX score & $16.2 \pm 7.6$ & $12.04 \pm 6.6$ & 0.003 \\
\hline Ejection fraction (\%) & $46.4 \pm 8.5$ & $49.8 \pm 6.5$ & 0.039 \\
\hline Endocan [ng/mL] & $2.41 \pm 0.48$ & $1.90 \pm 0.69$ & $<0.001$ \\
\hline Galectin-3 [ng/mL] & $5.25 \pm 1.06$ & $4.46 \pm 1.00$ & $<0.001$ \\
\hline
\end{tabular}

Continuous variables are presented as mean \pm SD and range (min-max). Categorical variables are presented as $n$ (\%). STR - ST-segment resolution; $\mathrm{BMI}$ - body mass index; HDL-C - high-density lipoprotein cholesterol; LDL-C - low-density lipoprotein cholesterol. 

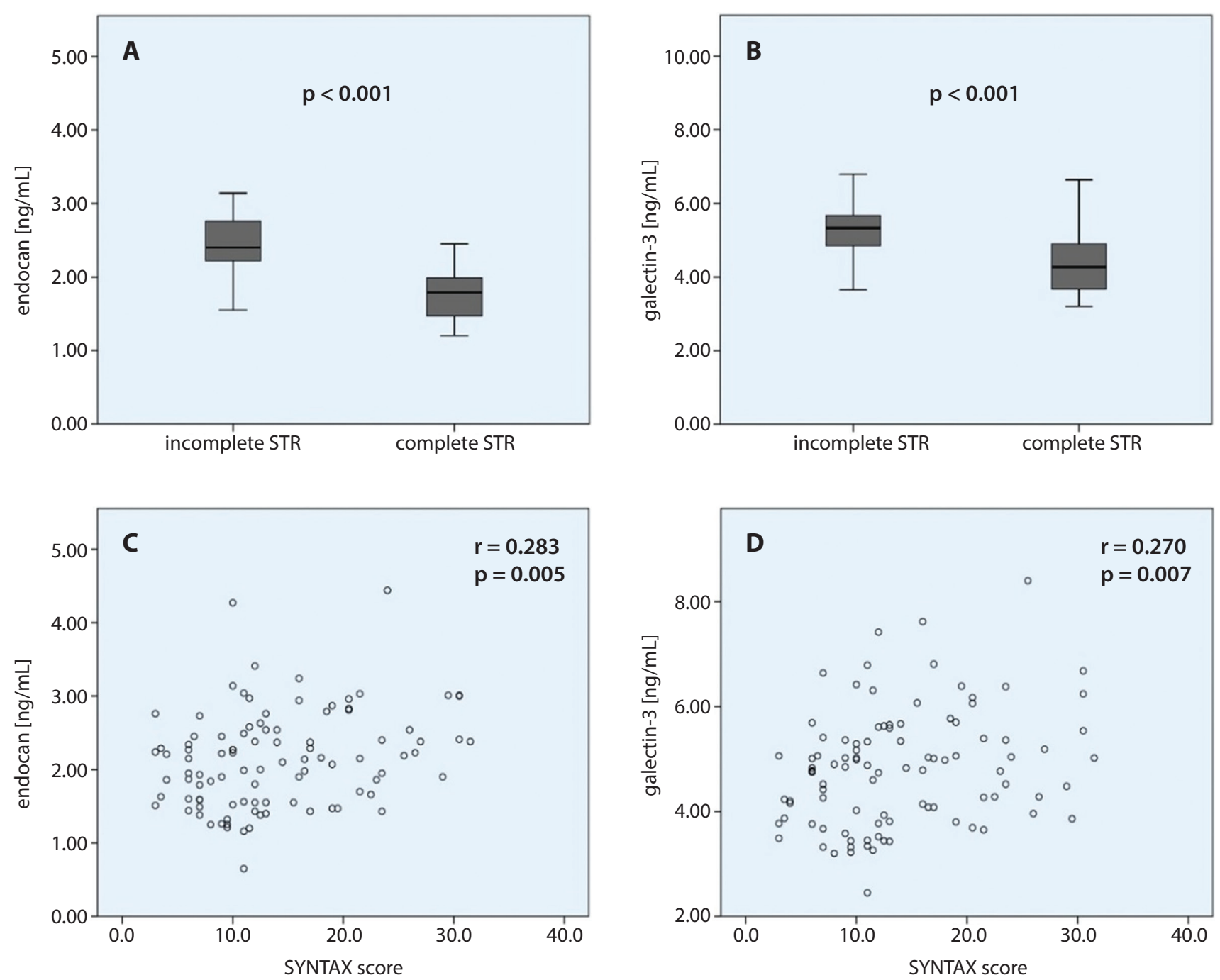

Fig. 1. A - comparison of endocan levels in the incomplete STR and complete STR groups; B - comparison of galectin-3 levels in the incomplete STR and complete STR groups; C - correlation between SYNTAX score and endocan; D - correlation between SYNTAX score and galectin-3

patients undergoing PPCI $(\mathrm{n}=98)$ were divided into a complete STR group $(\geq 70 \%, \mathrm{n}=53)$ and an incomplete STR group $(<70 \%, \mathrm{n}=45)$. Compared with the complete STR group, glucose, total cholesterol, low-density lipoprotein cholesterol (LDL-C), SYNTAX score, endocan and galectin-3 levels were significantly higher and the ejection fraction (EF) was significantly lower in the incomplete STR group ( $\mathrm{p}<0.05$ for all) (Fig. 1). The 2 groups were similar regarding age, gender, BMI, and CAD risk factors, such as arterial hypertension, diabetes mellitus, family history, and smoking ( $\mathrm{p} \geq 0.05$ for all).

Correlation analysis showed that the SYNTAX score was positively correlated with BMI $(r=0.227, p=0.025)$, peak troponin level $(\mathrm{r}=0.310, \mathrm{p}=0.03)$, endocan $(\mathrm{r}=0.283, \mathrm{p}=0.005)$, galectin $-3(\mathrm{r}=0.270, \mathrm{p}=0.007)$, and negatively correlated with EF $(\mathrm{r}=-0.145, \mathrm{p}=0.010)$ (Table 2) (Fig. 1).

Multivariate linear regression analysis revealed that BMI $(\beta=0.196, p=0.046)$ and galectin-3 $(\beta=0.212, p=0.037)$ were significantly associated with the SYNTAX score in patients with STEMI.
The results of multivariate logistic regression showed that in patients with STEMI, endocan (odds ratio $(\mathrm{OR})=0.406$; $95 \%$ confidence interval $(95 \% \mathrm{CI})=0.169-0.976 ; \mathrm{p}=0.044)$ and galectin-3 $(\mathrm{OR}=0.212 ; 95 \% \mathrm{CI}=0.084-2.752$; $\mathrm{p}=0.017$ ) were significant predictors of incomplete STR $(<70 \%)$ after PPCI.

\section{Discussion}

The main finding of the present study was that endocan and galectin-3 were independent predictors of incomplete STR (<70\%) after PPCI in patients with STEMI. Furthermore, along with BMI, galectin-3 was an independent predictor of the SYNTAX score.

Vascular inflammation is an important process in the initiation and progression of atherosclerosis. The inflammatory process affects the endothelial functions starting in the early phases of atherosclerosis. Endothelial dysfunction can be interpreted as the early step of cardiovascular disease development. Endocan and galectin-3 have 
Table 2. The correlation between SYNTAX score and clinical/demographic variables in STEMI patients

\begin{tabular}{|l|c|c|}
\multirow{2}{*}{\multicolumn{1}{c}{ Variables }} & \multicolumn{2}{|c|}{ SYNTAX score } \\
\cline { 2 - 3 } & $r$ & p-value \\
\hline Age & 0.188 & 0.064 \\
\hline BMI & 0.227 & 0.025 \\
\hline Waist circumference & 0.134 & 0.189 \\
\hline Heart rate & 0.131 & 0.197 \\
\hline Systolic blood pressure & -0.182 & 0.075 \\
\hline Diastolic blood pressure & -0.077 & 0.454 \\
\hline Initial troponin level & 0.093 & 0.370 \\
\hline Peak troponin level & 0.310 & 0.030 \\
\hline Serum glucose & 0.029 & 0.777 \\
\hline Serum creatinine & 0.112 & 0.279 \\
\hline Total cholesterol & 0.091 & 0.372 \\
\hline Triglyceride & 0.109 & 0.284 \\
\hline HDL-C & -0.130 & 0.203 \\
\hline LDL-C & 0.121 & 0.235 \\
\hline Ejection fraction & -0.145 & 0.010 \\
\hline Endocan & 0.283 & 0.005 \\
\hline Galectin-3 & 0.270 & 0.007 \\
\hline
\end{tabular}

SYNTAX - synergy between percutaneous coronary intervention with taxus and cardiac surgery; BMI - body mass index; $\mathrm{HDL}-\mathrm{C}$ - high-density lipoprotein cholesterol; LDL-C - low-density lipoprotein cholesterol.

a significant role in the regulation of the inflammatory process and may be interpreted as markers of endothelial activation. Galectin-3 plays an important role in both acute and chronic phases of inflammation by many immune reactions such as neutrophil activation, migration of the inflammatory cells and apoptosis. ${ }^{15}$ Endocan causes endothelial dysfunction by promoting adhesion molecules and migration of leukocytes across the damaged endothelium. ${ }^{16}$ Later in this cascade, activated adhesion molecules may also secrete chemokines, which are essential for inflammatory reaction and the ultimate outcome is the acceleration of the atherosclerotic process. ${ }^{17}$

There are individual studies suggesting that increased levels of endocan ${ }^{8}$ and galectin- $3^{18}$ are associated with CAD. In our study, which examined these biomarkers together, the results were consistent with the previous data. Although BMI, endocan and galectin-3 were significantly correlated with the SYNTAX score, only BMI and galectin-3 were independently associated with the SYNTAX score. Body mass index is reported to be a predictor of the extent and severity of coronary atherosclerosis. ${ }^{19}$ However, data about galectin-3 and SYNTAX score is relatively scarcer. Although both endocan and galectin-3 are associated with inflammation, galectin-3 has a complex relation with both acute and chronic phases of inflammation. Winter et al. suggested that galectin-3 was significantly related with premature myocardial infarctions, dyslipidemia and markers of inflammation. ${ }^{20}$ In this manner, galectin-3 may affect both plaque formation and plaque destabilization and this mechanism may explain the independent association of galectin-3 with the SYNTAX score. Galectin-3 may be interpreted as a novel biomarker that is independently associated with the complexity of coronary lesions.

The rupture of atherosclerotic plaque is the primary cause of STEMI. Through the complex mechanisms, including inflammation, oxidative stress and endothelial dysfunction, a cascade of events begins, leading to intimal thickening, development of plaque, eventually plaque rupture and clinical consequences. ${ }^{21}$ These mechanisms may also contribute to adverse outcomes during the follow-up of patients with a myocardial infarction. ${ }^{22}$

Two types of ischemia induced microvascular damage have been classified based on the pathophysiology. ${ }^{23}$ The first type is structural microvascular damage due to myocardial necrosis and the second type is functional microvascular damage in which the microvessels are intact and may be due to increased constriction of microvessels, edema, endothelial dysfunction or obstruction with platelets or neutrophils. Despite angiographically successful PPCI, lack of STR may be a good indicator of compromised tissue perfusion and more extensive myocardial damage.

As a novel finding, our results revealed that endocan and galectin-3 were independent predictors of incomplete STR in patients with STEMI. Besides the role of galectin-3 in inflammation, it also has effects on fibrosis and cardiac remodeling, thus galectin-3 is also reported to be associated with the development and worsening of heart failure. ${ }^{24}$ The prognostic role of galectin-3 in CAD is a current issue. In this manner, galectin-3 was reported to be an independent predictor of advanced heart failure and 30-day mortality in patients with STEMI. ${ }^{11,25}$ In a prospective study with 1013 CAD patients, Maiolino et al. reported that galectin-3 was a strong independent predictor of cardiovascular mortality. ${ }^{10}$ The relation of endocan and worse cardiovascular outcomes after STEMI was reported. ${ }^{8,26}$ Ye et al. demonstrated that serum endocan was associated with coronary slow flow. ${ }^{27}$ Qui et al. followed up 105 patients with STEMI for 3 months and reported that endocan was a predictor of major adverse cardiac events. ${ }^{28}$

Although the previous data indicates that both endocan and galectin-3 are valuable markers in predicting worse cardiovascular outcomes, to the best of our knowledge, there is no data concerning the association of STR, endocan and galectin-3 levels in STEMI patients. Considering the functional microvascular damage mechanism one can conclude that microvascular endothelial dysfunction and inflammation may explain the poor myocardial perfusion observed after PPCI. Although the pathophysiologic mechanisms by which higher levels of endocan and galectin-3 increase the risk of incomplete STR after PPCI are not clearly understood, possible hypothesis of our findings could be that elevated levels of endocan and galectin-3, as indicators of endothelial dysfunction and inflammation, 
may be surrogate markers of insufficient microvasculature perfusion at the myocyte level, which might contribute to incomplete STR.

The mechanism of the relationship between endocan, galectin- 3 and cardiovascular mortality and morbidity is still unclear. Considering the association between endocan, galectin-3, endothelial dysfunction and inflammation, it can be proposed that insufficient coronary reperfusion related with elevated endocan and galectin-3 may be a potential pathophysiological mechanism of the adverse cardiovascular outcomes in patients with STEMI.

Our study had some limitations; it was a cross-sectional study with a limited number of patients and there was no control group. It reflects a single center experience and in our laboratory, there was an upper limit for troponin results. The levels of endocan and galectin-3 were evaluated only at admission and they were not evaluated after the acute phase of the myocardial infarction. ST-segment resolution may be helpful, but it cannot be accepted as a definitive parameter for the microvascular impairment like the invasive hemodynamic procedures. Longitudinal studies may be designed to determine the long-term influence of these parameters on cardiovascular outcome.

\section{Conclusions}

To our knowledge, ours is the first report describing endocan and independent association of galectin-3 with incomplete STR in patients with STEMI. Based on the relation of endocan and galectin-3 with endothelial dysfunction, oxidative stress and inflammation, it can be proposed that insufficient coronary reperfusion related with elevated endocan and galectin-3 may be a potential pathophysiological mechanism of the adverse cardiovascular outcomes in patients with STEMI. The levels of endocan and galectin-3 may be helpful in identifying patients with a higher risk of insufficient myocardial perfusions and worse clinical outcome.

\section{ORCID iDs}

Yaşar Turan (1) https://orcid.org/0000-0002-2796-899X Vahit Demir (10 https://orcid.org/0000-0001-8349-6651

\section{References}

1. Davies MJ. The pathophysiology of acute coronary syndromes. Heart. 2000;83:361-366.

2. Carville S, Harker M, Henderson R, Gray H. Acute management of myocardial infarction with ST-segment elevation: Summary of NICE guidance. BMJ. 2013;347:f4006.

3. Ito $\mathrm{H}$, Okamura A, Iwakura $\mathrm{K}$, et al. Myocardial perfusion patterns related to thrombolysis in myocardial infarction perfusion grades after coronary angioplasty in patients with acute anterior wall myocardial infarction. Circulation. 1996;93:1993-1999.

4. Unikas R, Budrys P. Association between clinical parameters and STsegment resolution after primary percutaneous coronary intervention in patients with acute ST-segment elevation myocardial infarction. Medicina (Kaunas). 2016;52:156-162.

5. McLaughlin MG, Stone GW, Aymong E, et al. Prognostic utility of comparative methods for assessment of ST-segment resolution after primary angioplasty for acute myocardial infarction: The Controlled Abciximab and Device Investigation to Lower Late Angioplasty Complications (CADILLAC) trial. J Am Coll Cardiol. 2004;44:1215-1223.

6. Ozaki K, Toshikuni N, George J, et al. Serum endocan as a novel prognostic biomarker in patients with hepatocellular carcinoma. J Cancer. 2014;5:221-230.

7. Balta S, Mikhailidis DP, Demirkol S, et al. Endocan - a novel inflammatory indicator in newly diagnosed patients with hypertension: A pilot study. Angiology. 2014;65:773-777.

8. Kundi $H$, Balun A, Cicekcioglu $H$, et al. Admission endocan level may be a useful predictor for in-hospital mortality and coronary severity index in patients with st-segment elevation myocardial infarction. Angiology. 2017;68:46-51.

9. Martinez-Martinez E, Calvier L, Fernandez-Celis A, et al. Galectin-3 blockade inhibits cardiac inflammation and fibrosis in experimental hyperaldosteronism and hypertension. Hypertension. 2015;66:767-775.

10. Maiolino G, Rossitto G, Pedon L, et al. Galectin-3 predicts long-term cardiovascular death in high-risk patients with coronary artery disease. Arterioscler Thromb Vasc Biol.2015;35:725-732.

11. George M, Shanmugam E, Srivatsan V, et al. Value of pentraxin-3 and galectin-3 in acute coronary syndrome: A short-term prospective cohort study. Ther Adv Cardiovasc Dis. 2015;9:275-284.

12. Thygesen $K$, Alpert JS, Jaffe AS, et al. Fourth universal definition of myocardial infarction (2018). J Am Coll Cardiol. 2018;72:2231-2264.

13. Ong AT, Serruys PW, Mohr FW, et al. The SYNergy between percutaneous coronary intervention with TAXus and cardiac surgery (SYNTAX) study: Design, rationale, and run-in phase. Am Heart J. 2006;151:1194-1204.

14. Qiu CR, Fu Q, Sui J, et al. Serum endothelial cell-specific molecule 1 (Endocan) levels in patients with acute myocardial infarction and its clinical significance. Angiology. 2017;68:354-359.

15. Gucuk Ipek E, Akin Suljevic S, Kafes H, et al. Evaluation of galectin-3 levels in acute coronary syndrome. Ann Cardiol Angeiol (Paris). 2016; 65:26-30.

16. Lee W, Ku SK, Kim SW, Bae JS. Endocan elicits severe vascular inflammatory responses in vitro and in vivo. J Cell Physiol 2014;229:620-630.

17. Jaipersad AS, Lip GY, Silverman S, Shantsila E. The role of monocytes in angiogenesis and atherosclerosis. J Am Coll Cardiol. 2014;63:1-11.

18. Ozturk D, Celik O, Satilmis S, et al. Association between serum galectin-3 levels and coronary atherosclerosis and plaque burden/structure in patients with type 2 diabetes mellitus. Coron Artery Dis. 2015;26:396-401.

19. Zhang B, Pei C, Zhang $Y$, Sun $Y$, Meng S. High resting heart rate and high $\mathrm{BMI}$ predicted severe coronary atherosclerosis burden in patients with stable angina pectoris by SYNTAX Score. Angiology. 2018;69:380-386.

20. Winter MP, Wiesbauer F, Alimohammadi A, et al. Soluble galectin-3 is associated with premature myocardial infarction. Eur J Clin Invest. 2016;46:386-391.

21. Daiber A, Xia N, Steven S, et al. New therapeutic implications of endothelial nitric oxide synthase (eNOS) function/dysfunction in cardiovascular disease. Int J Mol Sci. 2019;20:187.

22. Zeller M, Korandji C, Guilland JC, et al. Impact of asymmetric dimethylarginine on mortality after acute myocardial infarction. Arterioscler Thromb Vasc Biol. 2008;28:954-960.

23. Galiuto L. Optimal therapeutic strategies in the setting of postinfarct no reflow: The need for a pathogenetic classification. Heart. 2004;90:123-125.

24. Salvagno GL, Pavan C. Prognostic biomarkers in acute coronary syndrome. Ann Transl Med. 2016;4:258.

25. Tsai TH, Sung PH, Chang LT, et al. Value and level of galectin-3 in acute myocardial infarction patients undergoing primary percutaneous coronary intervention. J Atheroscler Thromb. 2012;19:1073-1082.

26. Ziaee M, Mashayekhi S, Ghaffari S, Mahmoudi J, Sarbakhsh P, Garjani A. Predictive value of endocan based on TIMI risk score on major adverse cardiovascular events after acute coronary syndrome. Angiology. 2018:3319718815241.

27. Ye MF, Zhao ZW, Luo YK, Dong XF, Yan YM. Elevated endocan concentration is associated with coronary slow flow. Scand J Clin Lab Invest. 2016;76:345-348.

28. Qiu C, Sui J, Zhang Q, Wei P, Wang P, Fu Q. Relationship of endothelial cell-specific molecule 1 level in stress hyperglycemia patients with acute ST-segment elevation myocardial infarction: A pilot study. Angiology. 2016;67:829-834. 